\title{
IL CIRCOLO VIRTUOSO: SCIPIONE EMILIANO E I SUOI AMICI NELL'IMMAGINE DI CICERONE
}

\author{
Nota del m.e. GIANCARLO MAZZOLI (*)
}

(Adunanza dell's ottobre 2020)

SunTO. - L'immagine trasmessa da Cicerone specialmente nel De re publica e nel Laelius de amicitia ha alimentato la tesi tradizionale sul cosiddetto 'Circolo scipionico': il filellenismo, con più specifico riferimento ai nomi di Polibio e Panezio, avrebbe esercitato un profondo impatto a Roma già dalla metà del II sec. a.C.sui costumi, sulle idee e sulla vocazione filosofica del gruppo di aristocratici gravitante intorno alla figura carismatica di Scipione Emiliano. Tuttavia studi più recenti hanno fortemente messo in discussione se i processi culturali attivati dall'incontro col pensiero greco possano già essersi evoluti un secolo prima di Cicerone, entro una classe dirigente che assomiglia ancora troppo al ferus victor di Orazio; e anche Cicerone in altre opere rappresenta le principali figure di quel gruppo in modo meno idealizzato sul piano culturale, a partire dallo stesso Scipione e dal suo amico Lelio.

$$
* * *
$$

ABSTRACT. - The image transmitted by Cicero especially in the De re publica and in the Laelius de amicitia has fueled the traditional thesis on the so-called 'scipionic circle': philhellenism, with more specific reference to the names of Polybius and Panaetius, would have had a profound impact in Rome already from the middle of the second century B.C. on the customs, ideals and philosophical vocation of the group of aristocrats who gather around the charismatic figure of Scipio Aemilianus. However, more recent studies have strongly questioned whether the cultural process activated by the encounter with Greek thought could have already evolved a century before Cicero, within a ruling class that still too much resembles the Horatian ferus victor; and also Cicero in other works represents the main figures of that group in a less idealized way on a cultural level, starting with Scipio himself and his friend Laelius. 
L'epocale battaglia di Pidna che nel 168 a.C. chiude la terza guerra macedonica con la vittoria del console Lucio Emilio Paolo sul re Perseo è notoriamente l'evento dal quale si può far decorrere per Roma non solo l'egemonia sul mondo greco (poi materialmente sancita dalla distruzione di Corinto nel 146) ma anche una politica culturale via via più aperta in modo consapevole e recettivo verso quel mondo.

Dobbiamo fermarci sulla personalità del vincitore, figlio dell'omonimo console morto a Canne contro Annibale. Pur appartenendo all'incirca alla stessa generazione di Catone il Censore, Lucio Emilio Paolo è indubbiamente un personaggio per i tempi nuovi. Un tratto molto interessante concerne l'istruzione dei figli, curata da lui stesso, per ciò che riguarda i due di primo letto, anche dopo averli immessi, tramite l'adozione, in famiglie di primario rango e prestigio: uno, il maggiore, Quinto, nella gens Fabia, l'altro, il più giovane, nella famiglia degli Scipioni della gens Cornelia, col nome pertanto di Publio Cornelio Scipione Emiliano. Una educazione, la loro, in cui il rispetto della tradizione romana non impediva la vivace fruizione d'una formazione liberale ad ampio raggio, dalla passione per la caccia alla pittura alle lettere, con maestri venuti dalla Grecia appena vinta e anche coi libri del re Perseo, quasi unica preda bellica trattenuta in famiglia da Emilio Paolo, e appositamente per i figli, come attesta Plutarco (Aem. 28).

Tra gli arrivi dalla Grecia, particolare attenzione merita quello del grande storico Polibio di Megalopoli. Sebbene giunto, dopo Pidna, tra gli invisi ostaggi achei, sa subito mettere a profitto l'elevata posizione occupata nella patria greca per estrarsi dall'anonimato degli altri prigionieri, che finiscono dispersi tra le varie città italiche. Determinante per restare a Roma, e in posizione di tutto rispetto, è l'essere divenuto intrinseco di Scipione Emiliano, di lui più giovane d'una quindicina d'anni. E' Polibio stesso a raccontarci le cose nel memorabile excursus dedicato nel libro XXXI delle Storie (23-30) alla figura di Emiliano. Il legame tra i due nasce sì su basi culturali (per via d'un prestito di libri) ma prende subito una piega decisamente diversa. Illuminante un tratto del loro primo colloquio, in cui Polibio rassicura il ragazzo, che gli imputa di dedicare più attenzione al fratello maggiore, e si propone al suo fianco (XXXI 24, 5-8):

«per quanto riguarda me, volentieri mi metterei al tuo servizio e ti offrirei la mia collaborazione per aiutarti a parlare e agire in maniera degna dei tuoi antenati. Per quanto riguarda la formazione culturale, nella quale vedo che vi state ora impegnando seriamente, non vi mancheranno certo persone capaci di assistere con sollecitudine sia te che 
Fabio; di questi tempi vedo infatti affluire dalla Grecia una notevole folla di uomini che fanno al caso. Quanto invece al problema che ora, come tu affermi, ti affligge [...1, penso che tu non troveresti un alleato e un collaboratore più adatto di me» (trad. Sbardella).

La presa di posizione non potrebbe essere più chiara; e dovremo tenerne conto, a fronte dell'immagine che di Emiliano ci darà Cicerone. La collaborazione offerta dal Greco è tutta sul piano pragmatico, intesa a mettere la sua già larga competenza in campo politico e strategico al servizio del giovanissimo Scipione per favorirne l'ascesa pubblica. Polibio qui mostra di non attribuire preminente importanza ai $\mu \alpha \theta \eta \dot{\mu} \mu \alpha \alpha$ teorici, tra i quali non stentiamo a includere la filosofia: ne demanda la cura a qualche altro maestro greco, confermandone, da un lato, l'arrivo sempre più in forze nell'Urbe ma prendendone, dall'altro, nettamente le distanze. Polibio non è un filosofo ed è ben consapevole del contrastato trattamento riservato in quegli anni dell'immediato dopo-Pidna ai Greci che vengono a fare in Roma più aperta professione del 'mestiere' filosofico, guardati con sospetto negli ambienti più tradizionalistici e più volte addirittura banditi. Questo tocca specialmente a epicurei; ma anche la famosa ambasceria, nel 155, dei tre esponenti delle altre principali scuole greche, stoica, peripatetica, accademica, viene liquidata in fretta, per speciale pressione di Catone il Censore.

Il quadro prende a modificarsi solo a partire, all'incirca, dalla metà del secolo: possiamo, come data simbolica, assumere il 149, l'anno della morte, appunto, di Catone. Per la prima volta vediamo filosofi greci di professione ammessi a un rapporto più o meno diretto con importanti personaggi del mondo politico e culturale romano. L'approdo più saturo di conseguenze è quello dello stoico Panezio di Rodi e l'ambiente che lo accoglie è appunto quello che gravita intorno alla figura dell' ormai maturo Scipione Emiliano.

Pochi peraltro e vaghi sono i dati che possediamo sulla formazione del filosofo, così come sull'epoca stessa del suo arrivo a Roma. Sembra comunque che sia nato negli stessi anni di Scipione, dunque intorno al 185 a. C.: dato da tener presente, per evitare di accreditarlo al pari di Polibio, sulla scorta di talune tarde fonti, d'una auctoritas di precettore che mai egli ebbe nei confronti di Emiliano. Le testimonianze letterarie, e soprattutto quelle ciceroniane, mettono viceversa nel massimo risalto per lui lo stabilirsi d'un intimo rapporto di amicizia produttivo di vivaci effetti culturali. 
Leggiamo per esempio l'orazione pro Murena $(31,66)$ di Cicerone. L'avvocato invita Catone, il futuro Uticense, che è uno dei due accusatori del suo difeso, a temperare la propria intransigenza di severa impronta stoica, additandogli i ben più flessibili modelli offerti da Scipione e dai suoi amici, fin d'ora disegnati - siamo nell'anno consolare di Cicerone, il 63 - con un significativo profilo di bumanitas:

buiusce modi Scipio ille fuit quem non paenitebat facere idem quod tu, habere eruditissimum hominem Panaetium domi; cuius oratione et praeceptis, quamquam erant eadem ista quae te delectant, tamen asperior non est factus sed, ut accepi a senibus, lenissimus. Quis vero C. Laelio comior, quis iucundior <fuit> eodem ex studio isto, quis illo gravior, sapientior? Possum de L. Pbilo, de C. Gallo dicere baec eadem ${ }^{1}$.

Un evento che certamente contribuì non poco a cementare l'amicizia con Scipione è il viaggio di Panezio, unico non romano, al seguito dell' ambasceria compiuta da Emiliano in Oriente, e segnatamente in Egitto, più probabilmente tra il 140 e il 139 (se ne fa testimone, tra altri, ancora Cic. Ac. II 2, 5).

Panezio si fa portatore tra gli aristocratici romani della seconda metà del II sec. d'una visione filosofica che, se non confezionata su misura per loro, risultò comunque ben omologabile al loro ambiente $\mathrm{e}$ perciò atta, nella specifica congiuntura storica, a incontrarne il favore: uno stoicismo 'dal volto umano' che avverte molto, rispetto al rigorismo dei primi maestri, l'orma platonica e aristotelica e certo tiene conto delle serrate polemiche ingaggiate nei confronti dell'ortodossia scolastica in sede peripatetica e accademica. Per lui l'armoniosa bellezza del mondo dipende dalla stretta integrazione e interazione tra le sue parti e si rispecchia nell'organicità della natura umana, sussistente non solo nel complesso della struttura psicofisica ma anche al livello delle funzioni e delle responsabilità della vita associata.

1 «tale fu appunto il famoso Scipione, che era ben contento di comportarsi proprio come te tenendosi in casa quel gran dotto di Panezio»: Catone infatti aveva accolto presso di sé lo stoico Atenodoro di Tarso. «Fu la conversazione, furono gli insegnamenti di questo filosofo che, per quanto identici a quelli che ti stanno tanto a cuore, fecero di lui un uomo non già portato a un'eccessiva durezza, ma, com'ho sentito dire dai vecchi, straordinariamente umano. Chi d'altra parte fu più affabile di Lelio, chi più amabile pur appartenendo alla medesima tua scuola? E chi più di lui serio e saggio? Potrei dire altrettanto di Lucio Filo e di Gaio Gallo» (trad. Bellardi). 
Perché agire secondo virtù non è solo 'bene' ma 'bello': anche il microcosmo psichico, non meno che il macrocosmo fisico, è infatti oggetto per Panezio d'una percezione in termini estetici, fondata sulla dottrina del $\pi \rho \varepsilon ́ \pi o v$, del decorum. Va anzi detto che la scoperta dell'uomo come 'persona', $\mathrm{o}$, più precisamente, come composito, armonico insieme di entità 'personali' - nella piena valenza morale e sociale del termine e con tutta la pregnanza della metafora teatrale che gli inerisce - è una delle acquisizioni più rilevanti di cui Roma sia stata debitrice $\mathrm{a}$ Panezio. Ne darà sintetica illustrazione ancora Cicerone in off. I 30, 107; 32, 115, esponendo appunto la dottrina paneziana delle quattro persone che devono confluire unitariamente a costituire la 'personalità' di ciascun uomo:

\begin{abstract}
intellegendum etiam est duabus quasi nos a natura indutos esse personis, quarum una communis est ex eo, quod omnes participes sumus rationis praestantiaeque eius, qua antecellimus bestiis, a qua omne bonestum decorumque trabitur et ex qua ratio inveniendi officii exquiritur, altera autem quae proprie singulis est tributa. Ut enim in corporibus magnae dissimilitudines sunt, alios videmus velocitate ad cursum, alios viribus ad luctandum valere, itemque in formis aliis dignitatem inesse, aliis venustatem, sic in animis existunt maiores etiam varietates. [...] Ac duabus iis personis, quas supra dixi, tertia adiungitur, quam casus aliqui aut tempus imponit, quarta etiam, quam nobismet ipsis iudicio nostro accommodamus. Nam regna, imperia, nobilitatem, honores, divitiae, opes eaque, quae sunt his contraria, in casu sita temporibus gubernantur; ipsi autem gerere quam personam velimus, a nostra voluntate proficiscitur. Itaque se alii ad philosophiam, alii ad ius civile, alii ad eloquentiam applicant, ipsarumque virtutum in alia alius mavult excellere ${ }^{2}$.
\end{abstract}

2 «Bisogna capire anche che siamo stati rivestiti dalla natura, per così dire, di due maschere; una è comune, e deriva dal fatto che siamo tutti partecipi della ragione e della sua forza, con la quale siamo superiori alle bestie, dalla quale deriva ogni forma di moralità e di decenza e grazie alla quale riusciamo a trovare la via per definire la forma del dovere; l'altra è invece quella che è propriamente attribuita ai singoli. Come nei corpi esistono grandi differenze (vediamo alcuni prevalere nella velocità della corsa, altri nell'uso della forza per la lotta, e allo stesso modo in alcune forme si intravede l'equilibrio, in altre la bellezza), così a differenziare gli animi intervengono più grandi varietà [...] A quelle due maschere di cui ho parlato prima se ne aggiunge una terza, che le circostanze o i tempi impongono; e anche una quarta, che indossiamo secondo il nostro giudizio. Infatti i regni, i poteri, la nobiltà, le cariche, le ricchezze, i mezzi e tutto ciò che è contrario a queste cose, essendo in potere del caso, sono governati dalle circostanze; la maschera che invece noi stessi vogliamo portare deriva da una nostra scelta volontaria. Per questa ragione alcuni si dedicano alla filosofia, altri al diritto, altri ancora all'eloquenza, e ciascuno preferisce distinguersi in una particolare di queste attitudini» (trad. Marchese). 
In tal modo questo filosofo, lungi dal proporre, come i maestri vetero-stoici (Crisippo in particolare), lo scoraggiante e irraggiungibile modello del sapiens ideale, innesta la propria etica nella vita individuale e sociale di tutti i giorni, sostituendo all'azione morale assoluta ( $x \alpha \tau o ́ \rho \theta \omega \mu \alpha$, secondo il lessico stoico) quella relativa e 'conveniente' di volta in volta alle circostanze in cui si esercita: è il $x \alpha \theta \tilde{\text { qu}}$ Łov, cui viene dedi-

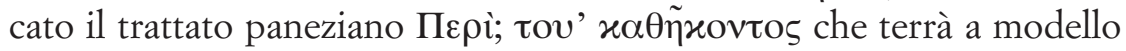
Cicerone per il suo de officiis. Per questa via - non senza cospicue innovazioni dottrinali che riguardano in particolare la rivalutazione della vita istintiva al fianco della razionalità- il pensatore perviene a incardinare il conseguimento del bene morale nel sistema delle quattro virtù cardinali. Ne troviamo il quadro sintetico sempre in Cicerone, ancora in off. I 5, 15:

\begin{abstract}
omne, quod est honestum, id quattuor partium oritur ex aliqua. Aut enim in perspicientia veri sollertiaque versatur aut in bominum societate tuenda tribuendoque suum cuique et rerum contractarum fide aut in animi excelsi atque invicti magnitudine ac robore aut in omnium, quae fiunt quaeque dicuntur ordine et modo, in quo inest modestia et temperantia. Quae quattuor quamquam inter se colligata atque implicata sunt, tamen ex singulis certa officiorum genera nascuntur?
\end{abstract}

Da questa rivisitazione delle quattro virtù stoiche (nell'ordine, in termini latini, prudentia, iustitia, fortitudo, temperantia) se da un lato esce ribadito il rilievo dato alla loro 'sistematica' interconnessione, dall'altro affiorano novità che ci aiutano a capire le ragioni del forte impatto esercitato da Panezio sugli ambienti aristocratici romani. In particolare la

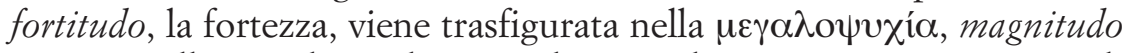
animi, quella grandezza d'animo che si traduce in un atteggiamento di superiorità nei confronti di tutte le contingenze esterne. E' la virtù del leader, inscindibile tuttavia dalla temperantia, mediante la quale il logos - il principio razionale al centro del sistema stoico - controlla gli impulsi

3 «La moralità nasce da ciascuna delle seguenti quattro parti: si colloca infatti nella disamina accurata della verità, oppure nella protezione accordata alla società umana nonché nell'attribuzione a ciascuno di ciò che gli spetta e nella garanzia degli accordi stipulati, oppure nella grandezza e nella forza di un animo eccelso e invitto, oppure nell'ordine e nella misura di tutte le cose che vengono dette e fatte, in cui risiede la moderazione e il controllo delle passioni. Sebbene queste quattro fonti siano tra loro collegate e intrecciate, tuttavia specifici tipi di dovere scaturiscono da ciascuna di esse» (trad. Marchese). 
istintivi garantendo la coerenza del carattere e dei comportamenti. Possiamo ben intuire quale personaggio potesse a Roma assurgere per Panezio a carismatico referente di questo plesso virtuoso. Per usare le parole di Max Pohlenz, autore del classico libro sullo Stoicismo in Grecia e a Roma4:

«Panezio, nel delineare la figura del capo, non ebbe davanti agli occhi i politici dei piccoli stati greci del suo tempo, ma gli uomini di stato romani che reggevano l'impero, e senza dubbio egli foggiò il suo ideale sul modello del grande che aveva potuto avvicinare personalmente. Alludiamo a Scipione, che egli propose esplicitamente come modello nella sua opera. Panezio vide in Scipione l'esempio di quella autoctisi di un'armonica personalità che gli appariva il naturale dovere dell'uomo; in lui trovò quella fusione di dignità intima ed esteriore che egli stesso, come aristocratico, perseguiva».

A sua volta Scipione Emiliano trova in Panezio (all'incirca suo coetaneo, come s'è detto) l'intellettuale greco da tenere al fianco in un rapporto di schietta familiarità. Si veda per es. come lo definisce Cicerone fin. IV 9, 23: bomo in primis ingenuus et gravis, dignus illa familiaritate Scipionis et Laelii. Il rapporto privilegiato con Scipione si estende infatti per via transitiva alla cerchia dei personaggi più strettamente legati al leader da vincoli di parentela, amicizia e comunanza politica, facendo di Panezio il punto più prestigioso di riferimento culturale per quasi tutti i membri di quel selezionato novero di aristocratici romani sul quale, a partire dalla metà dell' 800 in ambito tedesco (con Bernhardy, Hertz, Mommsen), è stata apposta la stereotipata etichetta di 'circolo scipionico'. La formula, come è ben noto, fa leva quasi per intero sulla testimonianza fornita a un secolo di distanza da Cicerone, soprattutto nei due dialoghi filosofici che eleggono ad ambiente quell'entourage, ritratto nell'anno 129, poco prima della morte di Scipione (nel grande De re publica) e poco dopo quella morte (nel breve Laelius de amicitia). Nel Laelius $(19,69)$ è Cicerone stesso ad applicare, per bocca del protagonista, cioè Lelio, l'amico per eccellenza di Scipione, la definizione di grex al gruppo dei suoi intimi:

4 M. Pohlenz, La Stoa: storia di un movimento spirituale, 1, trad. it., Firenze 1967, p. 413. 
maximum est in amicitia parem esse inferiori. Saepe enim excellentiae quaedam sunt, qualis erat Scipionis in nostro, ut ita dicam, grege. Numquam se ille Philo, numquam Rupilio, numquam Mummio anteposuit, numquam inferioris ordinis amicis, $Q$. vero Maximum fratrem, egregium virum omnino, sibi nequaquam parem, quod is anteibat aetate, tamquam superiorem colebat suosque omnes per se posse esse ampliores volebat 5 .

Nel De re publica il proemio al 1 . I pone come suggestivo preludio al vasto dibattito sulla costituzione statale il racconto del progressivo adunarsi di questo grex, alla spicciolata, come per una riunione di club, negli horti di Scipione all'inizio delle feriae Latinae (una festività in onore di Giove Laziale della durata di tre giorni, senza data fissa).

Cicerone sostiene (r. p. I 8, 13: ma il dato è da considerare senz'altro fittizio) di avere raccolto il resoconto dell'intero dialogo dalla viva voce d'uno dei partecipanti, Publio Rutilio Rufo, quando nel 78 (a ben oltre mezzo secolo di distanza dall'evento narrato) si recò a trovare questo fiero personaggio a Smirne, luogo d'esilio in cui volle restare sino alla fine dei suoi giorni dopo aver subìto l'ingiusta condanna per presunte malversazioni nei confronti dei provinciali. Primo a presentarsi da Scipione è il nipote (figlio della sorella Emilia) Quinto Elio Tuberone. Fiorisce affettuosa tra i due la conversazione, traendo spunto da un fenomeno meteorologico prodottosi in quei giorni (il parelio, o sdoppiamento dell'immagine solare): circostanza inquietante e giudicata ominosa (Scipione in effetti morrà di lì a poco in circostanze oscure). Per spiegare il fenomeno Scipione dice che ben volentieri sarebbe ricorso a Panezio, se non fosse stato solo idealmente presente, essendo nel frattempo tornato ad Atene per assumervi la guida della scuola stoica: quam vellem Panaetium nostrum nobiscum haberemus! $(10,15)$. L'incontro poi $(11,17)$ si arricchisce all'improvviso d'una nuova coppia, anch'essa accolta con viva cordialità: Lucio Furio Filo e, insieme, lo stesso narratore putativo del dialogo, Rutilio Rufo. E finalmente la brigata s'infoltisce quasi per aggregazione spon-

5 «Il massimo nell'amicizia è stare alla pari con gli inferiori. Spesso infatti c'è qualcuno che spicca, com'era Scipione nel nostro "gruppo". Lui non si mise mai al di sopra di Filo, di Rupilio, di Mummio o degli amici di rango inferiore; e, invece, siccome suo fratello Quinto Massimo - una persona eccellente, ma certo non al suo livello era più anziano, lo riveriva come un superiore e voleva che tutti i suoi amici fossero più importanti grazie a lui» (trad. Biddau). 
tanea con l'arrivo di cinque altre figure, in una animata sequenza narrativa che merita d'essere ricordata come una delle più belle 'cornici' dei dialoghi ciceroniani $(12,18)$ :

dixerat hoc ille, cum puer nuntiavit venire ad eum Laelium domoque iam exisse. Tum Scipio calceis et vestimentis sumptis e cubiculo est egressus, et cum paululum inambulavisset in porticu, Laelium advenientem salutavit et eos, qui una venerant, Spurium Mummium, quem in primis diligebat, et $C$. Fannium et Quintum Scaevolam, generos Laeli, doctos adulescentes, iam aetate quaestorios; quos cum omnis salutavisset, convertit se in porticu et coniecit in medium Laelium; fuit enim boc in amicitia quasi quoddam ius inter illos, ut militiae propter eximiam belli gloriam Africanum ut deum coleret Laelius, domi vicissim Laelium, quod aetate antecedebat, observaret in parentis loco Scipio. Dein cum essent perpauca inter se uno aut altero spatio conlocuti, Scipionique eorum adventus periucundus et pergratus fuisset, placitum est ut in aprico maxime pratuli loco, quod erat hibernum tempus anni, considerent; quod cum facere vellent, intervenit vir prudens omnibusque illis et iucundus et carus, M'. Manilius qui a Scipione ceterisque amicissime consalutatus adsedit proximus Laelio ${ }^{6}$.

La coreografia della vivace scena reca certo la firma di Cicerone; eppure, al netto dell'idealizzazione, sembra ritrarre in modo plausibile i giochi di forze e lo spirito dei rapporti interpersonali effettivamente vigenti nel gruppo: che presenta davvero sul piano visivo un carattere di 'circolarità' con quella posizione centrale occupata vicendevolmente domi bellique da Scipione e da Lelio e con quel muoversi in giro dei personaggi che sembra obbedire alle istanze preliminari d'una reciproca

6 «Aveva egli finito di dir ciò, quando il giovane servo annunciò che stava venendo da lui Lelio, ed era già uscito di casa. Scipione allora, messosi calzari e abiti, uscì dalla camera da letto e, dopo aver passeggiato un poco nel portico, salutò Lelio che giungeva e quelli che erano venuti con lui, Spurio Mummio, che aveva caro tra i primi, e Gaio Fannio e Quinto Scevola, generi di Lelio, giovani colti e in età ormai di ex questori. Dopo averli salutati tutti, si girò nel portico e fece mettere in mezzo Lelio: nella loro amicizia c'era infatti questo, quasi un reciproco impegno formale, che al campo Lelio riverisse l'Africano quale un dio per la sua insigne gloria in guerra; in città, viceversa, Scipione onorasse Lelio come un padre, perché lo avanzava d'età. Quindi, dopo essersi scambiate pochissime parole, muovendosi per uno o due giri - e la loro venuta era riuscita tanto gradita e piacevole a Scipione - si decise di andare a sedere nel posto più soleggiato del praticello, perché era la stagione invernale; quando si disponevano a muoversi, sopraggiunse il giureconsulto Manio Manilio, a tutti loro gradito e caro, il quale, salutato in coro con viva cordialità da Scipione e da tutti gli altri, si sedette vicinissimo a Lelio» (trad. Cancelli). 
presa di misure. Aleggiano, sia pure in absentia, i referenti intellettuali di Panezio e di Polibio, ricordati più oltre insieme nel corso del dialogo. È Lelio infatti a evocarli in dibattito con Scipione, in merito alla costituzione romana (I 21, 34):

memineram persaepe te cum Panaetio disserere solitum coram Polybio, duobus Graecis vel peritissimis rerum civilium, multaque colligere ac docere, optimum longe statum civitatis esse eum quem maiores nostri nobis reliquissent ${ }^{\top}$.

Due dunque sono le generazioni che s'aggregano nel giardino di Scipione: da una parte quella dei senes, rappresentata dallo stesso Emiliano, dal grande amico, di poco più anziano, Lelio, antonomastico Sapiens, e inoltre da aequales come Filo, Mummio e Manilio; dall'altra quella di personaggi presentati come ancora adulescentes nel 129, quali Tuberone, Rutilio e i due generi di Lelio, Fannio e (in realà alquanto più anziano) Quinto Mucio Scevola l'Augure: sono i due stessi cui Cicerone farà intrattenere col suocero di lì a poco il dialogo De amicitia, intestato al nome di Lelio, 1'amico' per eccellenza, e raccontato a Cicerone, nella finzione della cornice, proprio da Scevola l'Augure $(1,3)$.

E' sul ruolo culturale di tutte queste figure (e di poche altre assenti dal bel quadretto per ragioni cronologiche) che s'incentra il problema della valenza filosofica del cosiddetto 'circolo' scipionico.

La tesi tradizionale, conferendo molto peso alla portata storica delle testimonianze ciceroniane, è propensa non solo ad attribuire consistenza alla caratura filosofica delle singole personalità (in primis della coppia centrale, Scipione e Lelio) ma anche a dare il massimo risalto alla coesione 'circolare' e all'egemonia intellettuale che l'ambiente scipionico avrebbe dimostrate a Roma nel corso dell'intero II secolo ${ }^{8}$. L'incontro col pensiero greco, con riferimento specifico ai nomi di Polibio e Pane-

7 «Ricordavo che tu eri solito discuterne molto spesso con Panezio davanti a Polibio, due tra i Greci più esperti in materia politica, pervenendo sulla scorta di molti argomenti a insegnare che la costituzione lasciataci in eredità dai nostri antenati è di gran lunga la migliore» (trad. Cancelli).

8 Cf. R.M. Brown, A Study in the Scipionic Circle, «Iowa Studies in Classical Philology», I, 1934; J.M. ANDRÉ, L'otium dans la vie morale et intellectuelle romaine des origines à l'époque augustéenne, 1966, pp. 135-201; P. GRIMAL, Il secolo degli Scipioni, trad. it., Brescia 1981. 
zio, avrebbe fin da allora segnato in profondità i costumi, gli ideali e le stesse pratiche culturali dell'aristocrazia romana, nella luce d'una nuova spiritualità che, senza rinnegare i valori più consacrati del mos maiorum, avrebbe tratto dal filellenismo l'alimento più ricco di cui nutrire il suo valore specifico, cioè il sentimento e l'esercizio dell'bumanitas. Ora, tutto ciò è innegabile in una prospettiva di più lungo termine, che troverà nei dialoghi ciceroniani il suo monumentum aere perennius; ma riesce ben difficile assumere quei dotti conversari tra gentiluomini proprio come documenti d'un processo culturale che si presentava già evoluto un secolo prima, in seno a una classe dirigente ancora troppo simile al ferus victor di oraziana memoria, impegnata com'era a finir di assicurare a Roma la supremazia nel mondo mediterraneo con conquiste anche brutali. Anzi, proprio Scipione Emiliano si era assunto il compito nel $146 \mathrm{di}$ eliminare per sempre il problema Cartagine radendola totalmente al suolo, salvo poi essere accreditato dalla tradizione di edificanti, o quanto meno pensose, lacrime davanti alla città in fiamme da lui stesso fatte appiccare; e non basta: nel 133, solo quattro anni prima delle illuminate conversazioni raccontate da Cicerone nel De re publica, era stato dietro le quinte dell'assassinio di Tiberio Gracco e aveva preso per fame la città iberica di Numanzia dopo un assedio così feroce da costringere i disgraziati abitanti a gesti disperati. È il caso, del resto, di tornare a ricordare le peculiarità dell'impegno educativo assunto nei suoi confronti da Polibio, mirato a ben altro che a impartirgli una cultura filosofica; piuttosto, a gettare le basi del suo prestigio politico.

Da più parti, in effetti, in tempi più recenti, la tesi tradizionale sul 'circolo' è stata utilmente revocata in discussione'. Particolarmente incisive sono state le critiche di Hermann Strasburger ${ }^{10}$, che ha posto a confronto gli splendidi ritratti di Scipione e Lelio offerti nel De re publica e nel Laelius con altre, più realistiche, valutazioni. Lo stesso Cicerone, si badi, non evita i toni chiaroscurali quando nel Brutus $(21,83)$ scende a giudicare in concreto il loro stile:

de ipsius Laeli et Scipionis ingenio quamquam ea est fama, ut plurimum tribuatur ambobus, dicendi tamen laus est in Laelio inlustrior. at oratio Laeli de collegiis non melior quam de multis quam voles Scipionis; non

9 Cf. G. GARBARINO, Roma e la filosofia greca dalle origini alla fine del 2. secolo a. C., raccolta di testi con introduzione e commento, Torino 1973, I, pp. 14-49.

10 H. Strasburger, Der 'Scipionenkreis', in «Hermes» 94, 1966, pp. 60-72. 
quo illa Laeli quicquam sit dulcius aut quo de religione dici possit angustius, sed multo tamen vetustior et horridior ille quam Scipio ${ }^{11}$.

Nel focalizzare sul piano retorico i suoi giudizi, Cicerone si guarda bene dall'incorrere in anacronismi o idealizzazioni, dedicando alle due figure di punta del 'circolo' profili meno smaglianti, con apprezzamenti tutt'altro che assoluti. Non è certo il loro prestigio morale o politico a uscirne intaccato ma l'effettivo tenore della formazione culturale, con (ciò che a noi più interessa) un argumentum e silentio relativo al grado di compenetrazione raggiunto in età matura nelle conoscenze filosofiche (con specifico riguardo al pensiero stoico). E' questo un tema sul quale l'autore del Brutus si mantiene del tutto reticente, non meno che il Polibio del, pur magnifico, medaglione dedicato a Emiliano: facendo nell'ultima parte di questo (XXXI 28, 10-29, 12) il bilancio sul personaggio, lo storico greco altro non gli accredita se non il pieno

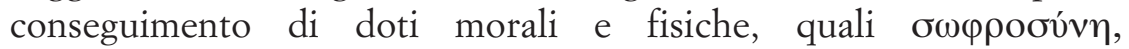

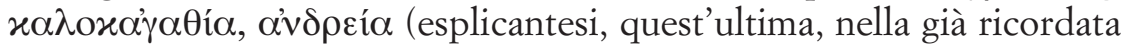
passione per la caccia). D'altra parte, nelle idee attribuite ai protagonisti del 'circolo' nel De re publica e nel Laelius va senz'altro colta, in certa misura, la lezione di Polibio stesso e, probabilmente, anche di Panezio: non si può dunque tacciare d'inverosimiglianza Cicerone per aver conferito già lo sfondo del II secolo a problematiche politiche e sociali su cui i due pensatori greci hanno avuto il merito di sensibilizzare per la prima volta l'aristocrazia romana; ma sarebbe arbitrario passare da questa ammissione, senza specifiche e circostanziate convalide, al ritenere che l'ambiente scipionico le abbia, per così dire, metabolizzate in proprio e consegnate alle generazioni successive; sicché non si può dare torto allo Strasburger quando ${ }^{12}$ giunge a definire «eine gigantische Paradoxie» la pretesa di attribuire a Emiliano - sulla falsariga ciceroniana già dell'orazione Verrina de signis del 70 (II 4, 98) - le qualità superlative di homo doctissimus atque humanissimus.

11 «Per ciò che riguarda l'ingegno di Lelio e di Scipione, sebbene tale ne sia la fama, che ambedue godono di un altissimo apprezzamento, nell'eloquenza è tuttavia più insigne la reputazione di Lelio. Eppure l'orazione di Lelio sui collegi non è migliore di una qualsiasi tra le molte di Scipione; non perché vi sia qualcosa di più gradevole di quel celebre discorso di Lelio, o perché della religione si possa parlare in termini più augusti; tuttavia egli è molto più vetusto e ruvido di Scipione» (trad. Narducci).

12 H. STRASBURger, Der 'Scipionenkreis', cit., p. 69. 
Lo stesso Cicerone, nel prosieguo del passo del Brutus prima citato $(21,84)$, è costretto a far pendere rispetto a lui la bilancia dell'ingegno e della cultura letteraria dalla parte di Lelio; ma la personalità di quest'ultimo, proprio per il ruolo complementare esercitato, e da Cicerone tanto enfatizzato, nei confronti di Scipione, non riesce a sollevarsi dal rango, nel complesso umbratile, di paredro del grande leader. E del resto la sua stessa qualità antonomastica, la sapientia, che Cicerone, com'era da attendersi, dichiara nel trattato de amicitia fondata non solum natura et moribus, verum etiam studio et doctrina (Lael. 2, 7), viene presso altri interpreti antichi (Lucilio, Plutarco) ricondotta in vario modo, più realisticamente, alla sfera pratica «Lelio - ha osservato J.M. André13 illustra con la sua sapientia un robusto senso concreto, la volontà d'applicare cultura e intelligenza all'analisi della situazione politica», dimostrando un atteggiamento che, sul piano storico, si traduce anche per lui in contatto più coi pensatori (pure nel suo caso specialmente con Panezio) che col loro pensiero: considerazioni che si possono ancor più facilmente estendere ai personaggi comprimari del 'circolo', tutti in primis a vario titolo impegnati molto concretamente in quel cruciale periodo nella gestione del potere.

Nemmeno al loro riguardo il Brutus esprime in genere apprezzamento sul piano retorico; e, per ciò che concerne il rapporto con la filosofia, fornisce indicazioni positive solo sulle figure dell'ultima generazione - Fannio, Scevola, Tuberone, Rutilio - che la cronologia consente effettivamente di annoverare tra gli auditores latini di Panezio. Nel Brutus Scevola, pur accreditato di competenza giuridica e prudentia, viene escluso dal novero degli oratori $(26,102)$; circa gli altri tre, è significativo che il giudizio più o meno drasticamente sfavorevole sulla loro eloquentia, con uno scarto netto rispetto al piano delle facoltà dialettiche, venga fatto discendere proprio dall'adesione allo stoicismo $(31,118)$, dottrina che poco indulge agli ornamenti del parlar bene.

Ciò che sembra dunque opportuno, rispetto all'opinione più tradizionale, non è certo mettere in dubbio la circolazione, attorno al carismatico leader, d'una élite accomunata da ragioni familiari e politiche e anche dal favore accordato ad alcuni cospicui esponenti del pensiero greco; bensì ridimensionare da un lato il ruolo attribuito agli interessi intellettuali e filosofici come principale legante del gruppo, dall'altro la

13 J.M. ANDRÉ, L'otium, cit.,, p. 184, nota 9. 
propensione a fare dell'apertura al mondo greco nel maturo secondo secolo una marca esclusiva di Scipione e dei suoi amici. Su quest'ultimo punto non si possono che condividere le prudenti e documentate riserve espresse da alcuni studiosi (come l'Astin e il Jocelyn ${ }^{14}$ ), riconoscendo tendenze (talora smodatamente) filelleniche pure in personaggi che nulla hanno a che vedere con l'ambiente scipionico o addirittura si segnalano per ostilità nei confronti di qualche suo esponente.

In ultima analisi, non abbiamo dunque concreti elementi per far decorrere dall'età scipionica i primi svolgimenti del pensiero filosofico tra i Romani: anche per le figure più segnate dall'impronta stoica non troviamo documentabili ricadute sul piano dottrinale, marcando essenzialmente quell'impronta una scelta di vita, un fatto di decorum morale e intellettuale. Pensiamo ancora ai giudizi del Brutus su Tuberone e su Rutilio Rufo, il protomartire repubblicano. Per il primo, che pur fu destinatario di scritti di Panezio e del suo discepolo Ecatone, non abbiamo tracce positive di una personale produzione letteraria, tanto meno filosofica; del secondo c'è sì attestata, al contrario, un'ampia attività (soprattutto di carattere oratorio e memorialistico, al tempo dell'intransigente esilio), ma senza alcun diretto rapporto con la sua opzione stoica $^{15}$. E va peraltro detto - alla fine dei conti - che è Cicerone stesso a giudicare motivata l'assenza d'una specifica letteratura filosofica latina di estrazione 'scipionica'. Ecco come si esprime in Tusc. IV 3, 5 s.:

sapientiae studium vetus id quidem in nostris, sed tamen ante Laeli aetatem et Scipionis non reperio quos appellare possim nominatim. Quibus adulescentibus Stoicum Diogenem et Academicum Carneadem video ad senatum ab Atheniensibus missos esse legatos, qui cum rei publicae nullam umquam partem attigissent essetque eorum alter Cyrenaeus alter Babylonius, numquam profecto scholis essent excitati neque ad illud munus electi, nisi in quibusdam principibus temporibus illis fuissent studia doctrinae. Qui cum cetera litteris mandarent, alii ius civile, alii orationes suas, alii monumenta maiorum, hanc amplissimam omnium artium, bene vivendi disciplinam, vita magis quam litteris persecuti sunt. Itaque illius verae elegantisque philosophiae, quae ducta a Socrate in Peripateticis

14 A.E. Astin, Scipio Aemilianus, Oxford 1967, pp. 294-296; H.D. Jocelyn, The Ruling Class of the Roman Republic and Greek Philosophers, in «Bull. John Rylands Libr.» 59, 1977, pp. 323-366: 327.

15 Cf. G. MoretTI, Suscitare o no le passioni? Il ruolo di Publio Rutilio Rufo, in L. Calboli Montefusco (ed.), Papers on Rherotic, IV, Roma 2002, pp. 205-222. 


\section{adbuc permansit et idem alio modo dicentibus Stoicis, cum Academici eorum controversias disceptarent, nulla fere sunt: aut pauca admodum Latina monumenta sive propter magnitudinem rerum occupationemque hominum, sive etiam quod imperitis ea probari posse non arbitrabantur ${ }^{16}$.}

Ben si vede come - a parte il riconoscimento d'un interesse, una buona disposizione verso le dottrine di matrice greca - nemmeno l'età scipionica esca risparmiata dal giudizio di Cicerone, che investe anzi l'intero periodo alle sue spalle per rivendicare una orgogliosa primazia per quanto attiene a una scrittura filosofica latina provvista di dignità letteraria. In un periodo in cui le prevenzioni contro la cultura greca vanno su vari fronti attenuandosi, Emiliano e il suo entourage aristocratico si collocano sulle linee avanzate dell'apertura, traendo a loro volta dagli apporti dottrinali ellenici (con particolare riguardo al rinnovato sistema delle virtù) le 'decorose' patenti per il proprio operare in pubblico e in privato: appunto, in definitiva, un 'circolo virtuoso' tra Roma e Grecia, atto a presentarsi, nel secolo successivo, come locus ideale per le più nostalgiche proiezioni del pensiero ciceroniano.

16 «L'interesse per la sapienza è certamente antico presso di noi, però non riesco a trovare nomi da citare per il periodo anteriore a Lelio e a Scipione. Quando questi erano giovani, mi risulta che furono mandati dagli Ateniesi come ambasciatori presso il senato lo stoico Diogene e l'accademico Carneade»: è l'accennata famosa ambasceria del 155; «essi non si erano mai occupati di politica, uno era di Cirene e l'altro babilonese: certamente non sarebbero stati tolti al loro insegnamento e scelti per quell'incarico, se in quei tempi certi nostri personaggi in vista non avessero dimostrato interesse per la cultura filosofica. Essi però affidavano allo scritto gli altri loro studi, chi il diritto civile, chi i propri discorsi, chi le memorie degli antenati; ma preferirono attendere a questa dottrina, che insegna a vivere bene ed è la più nobile di tutte le arti, con la loro vita più che con i loro scritti. Pertanto quella vera e ottima filosofia che, iniziata da Socrate, ebbe continuatori fino ad oggi nei Peripatetici, ed anche negli Stoici che sostengono le stesse idee in modo diverso, mentre gli Accademici fanno da arbitri nelle loro controversie, non è rappresentata da quasi nessuna o da ben poche opere in latino, sia perché l'impresa era grande e gli uomini troppo affaccendati, sia anche perché pensavano che tali studi non potevano essere apprezzati da gente del tutto profana» (trad. Marinone). 
